\title{
Size perception is less context-sensitive in males
}

\author{
William A Phillips, Katie L S Chapman, P Daniel Berry \\ Centre for Cognitive and Computational Neuroscience, Department of Psychology, University of Stirling, \\ Stirling FK94LA, Scotland, UK; e-mail: Wap1@stir.ac.uk \\ Received 16 June 2003, in revised form 2 October 2003
}

\begin{abstract}
Context sensitivity of size perception has previously been used to study individual differences related to the distinction between local, analytic, or field-independent and global, holistic, or field-dependent perceptual styles. For example, it has been used in several recent studies of autistic spectrum disorders, which may involve an excessive bias toward local processing. Autism is much more common in males, and there is evidence that this may be in part because males in general tend to be less context-sensitive than females, and thus are more affected by conditions that further reduce context sensitivity. There is also evidence that a bias to local processing is more common in professions that require attention to detail. Context sensitivity of size perception was therefore studied as a function of sex and academic discipline in sixtyfour university staff and students by a simple, sensitive, and specific psychophysical measure based on the Ebbinghaus illusion. The results show that in this task males are on average less context-sensitive than females, that the overlap is large, and that subjects with very high or very low context sensitivity tend to have the sex and profession predicted by the above hypotheses.
\end{abstract}

\section{Introduction}

Contextual interactions are a central component of cognition in general and perception in particular. Much is known about their anatomical and physiological bases (Phillips and Singer 1997), and there is now even a realistic possibility of relating them to wellspecified mechanisms of synaptic interaction, and to psychopathologies that involve cognitive disorganisation. For in-depth discussions of this possibility see Phillips and Silverstein (2003) and the associated commentaries and response. Studies of context sensitivity in vision therefore have potentially broad relevance to both normal and abnormal perception.

Extensive discussion of the concept of context is provided by Phillips and Silverstein (2003) and by Phillips and Singer (1997), but in short we think of context as activity that affects the processing of signals without changing what they mean, ie what they transmit information about. Thus, contextual interactions are a special class of interactions that do not themselves drive activity but which modulate the effects of those that do. Context is therefore neither necessary nor sufficient to produce the responses observed, but it can modulate the effects of conditions that are sufficient.

Context sensitivity is currently much discussed as a major component of individual differences in cognitive style. For example, it has been proposed that there are two broad cognitive styles: one that is context-insensitive, local, analytic, and field-independent; and one that is context-sensitive, global, holistic, and field-dependent (Happé 1999). There is evidence that males are less context-sensitive than females (Baron-Cohen 2002; Baron-Cohen et al 2003), and this includes work done several decades ago in which the rod-and-frame test was used to study the effects of tilted frames, etc, on the perception of the vertical where males were reported to be less context-sensitive than females (Witkin et al 1954). This finding has been replicated by others, but it is not wholly reliable, and the conditions under which it occurs are not yet well understood (Voyer et al 1995). Differences between males and females have also been studied with the use of many other spatial tasks. For some tasks they seem to be found more often 
than not, but for others reported differences are unreliable [see Voyer et al (1995) for an extensive review]. Even where differences are found, a major problem is that we do not know what specific aspects of spatial processing were being measured, as many different processes contribute to performance of the tasks that were used (Voyer et al 1995). We address this problem here by using a paradigm that provides an unequivocal and specific measure of context sensitivity.

Further evidence that males tend to be less context-sensitive than females is that autistic subjects seem to have very low context sensitivity, and they are much more likely to be male than female. Findings from various tasks show that autistic subjects are biased towards a form of processing that is excessively local, or with 'weak central coherence', and prominent amongst these are the tasks designed to measure the effect of context on visual size perception (Happé 1999). For example, Happé (1996) showed autistic subjects various illusions, including the Ebbinghaus illusion, and concluded that they were not susceptible to them. This implies that size perception in autistic subjects is not sensitive to context. These findings are still much debated, however, so the extent of any such insensitivity is not yet clear (eg Ropar and Mitchell 1999). If the context sensitivity of size perception in autistic subjects is low, however, and, if this is an extreme form of a perceptual style that is more common in males, then size perception will in general be less context-sensitive in males than females. We test this prediction here using a specific and sensitive psychophysical test based upon the Ebbinghaus illusion (figure 1).

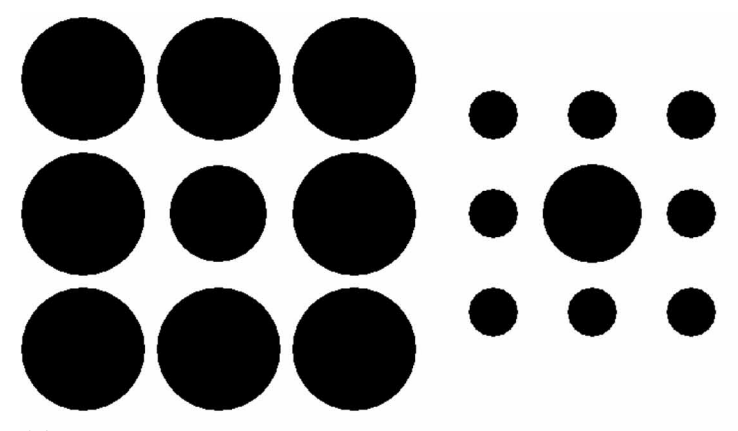

(a)

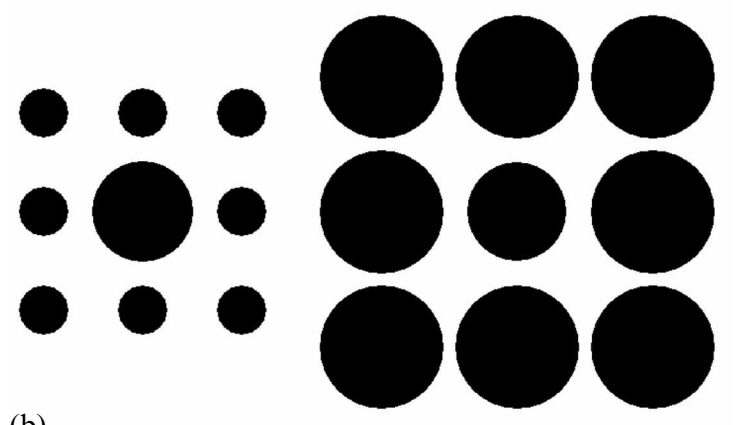

Figure 1. Two examples of the stimulus displays used. In both, the centre circle on the right is $2 \%$ larger than that on the left. In the top display the larger centre circle is in small surrounds; this makes the difference easy to see. In the bottom display the larger centre circle is in even larger surrounds. This makes the real difference hard to see, and can make the centre circle on the left seem larger than that on the right.

(b)

Happé et al (2001) studied the context sensitivity of size perception in the parents of autistic boys, and in a group of matched control subjects, but the findings in relation to sex differences were equivocal. Fathers of autistic boys had low context sensitivity, but their mothers did not. This is evidence for a difference in the predicted direction, but in contrast to this there was no evidence of any difference between males and females in the control subjects. One possible reason for this ambiguity is that the method used to assess the effects of context on size perception had low specificity and low sensitivity. 
Specificity was low because response bias to say that the sizes did not differ was uncontrolled, and use of the size of the surrounds to determine response was not controlled. Sensitivity of the measure used was low because the estimate of context sensitivity was based on only 6 trials per subject. One goal of the work reported here was to develop an experimental paradigm that had higher specificity and sensitivity, but which was still simple enough to be used with a wide range of subjects.

There is also evidence that individual differences in cognitive style are associated with differences in real-life interests and occupations. For example, Briskman et al (2001) found that the fathers of autistic boys were more likely to be employed in 'detail focused' occupations such as computing and accountancy than control subjects. BaronCohen et al (2001) developed a self-report questionnaire designed to assess individual differences in such processes, and found that scientists and mathematicians scored significantly higher than students of the humanities or social sciences. We therefore studied staff and students in the Departments of Computing Science and Mathematics using our performance-based psychophysical measure of context sensitivity, and compared them with staff and students in the Department of Applied Social Science. Both staff and students were studied because of the possibility that an academic career may in general put a greater emphasis upon attention to detail than do most other professions.

\section{Method}

The specific paradigm we used was developed through several prior studies that aimed to produce a measure specific to the effects of context, sensitive to individual differences, easy for subjects to understand, applicable directly to the general population without any prior parametric adjustment, requiring only a few minutes of subject time, and simple enough to use with autistic subjects and others with a learning disability. The paradigm we used meets these aims.

A two-alternative forced-choice method was used in which the task was to decide whether the larger centre circle was on the left or on the right, and to indicate that by pressing either the left-pointing or the right-pointing arrow on the keyboard.

\subsection{Subjects}

For our study a total of sixty-four subjects were recruited on the basis of their availability and willingness to participate. They received no payment. There were eight subjects in each of eight groups specified by all possible combinations of sex, academic status (staff or student), and academic discipline (computing science and mathematics or applied social science). Approval for the study was obtained from the Ethics Committee of the Psychology Department of Stirling University before recruiting subjects.

\subsection{Stimuli and design}

On each trial two $3 \times 3$ arrays of circles were presented, one to the left and one to the right of fixation (figure 1). The centre circle of one array was 100 screen pixels in diameter. The centre circle of the other was 82, 86, 90, 94, 98, 102, 106, 110, 114, or 118 pixels in diameter. Each of these ten size differences was presented eight times with the larger centre circle surrounded by circles 125 pixels in diameter, and the smaller centre circle surrounded by circles 50 pixels in diameter. In these conditions size contrast impairs discrimination. To unconfound surround size and centre size the 98 and 102 pixel conditions were presented eight times each with the smaller centre circle surrounded by circles 125 pixels in diameter, and the larger centre circle surrounded by circles 50 pixels in diameter. Size contrast then increases accuracy, if subjects are judging the apparent sizes of the centre circles. These 96 trials $[(10 \times 8)+(2 \times 8)]$ were presented in random order. On each trial the two arrays were presented for $2000 \mathrm{~ms}$. 


\subsection{Apparatus}

A specially developed and flexible program running in Java was used to compute all parameters for each trial, to control presentation, and to record and analyse responses. A Toshiba 4090 CDS laptop was used, with a 13-inch LCD/TFT monitor, and 800 by 600 pixel resolution.

\subsection{Procedure}

Subjects were tested singly in a quiet secluded room. They were shown an example of the stimulus arrays to be used and the task was explained. They were told to ignore the surrounding circles and to compare the sizes of the centre circles. If they thought that the one on the left was larger, then press the left-pointing arrow key; if on the right, then press the right-pointing arrow key. They were told that the two circles would never be the same size, and that, if they were not sure, then to make the best guess that they could. Subsequent trials followed automatically after between about 1000 and $3000 \mathrm{~ms}$ after response. The distance between the subject and the screen was that normal for laptop operation. The whole experiment took about 7 min per subject. No feedback was given concerning the accuracy of response.

\section{Results}

The context conditions of central interest in this study were those in which the larger centre circle was surrounded by even larger circles and the smaller by even smaller ones. Size contrast then reduces the accuracy of discrimination. However, subjects could always be correct in these conditions, by ignoring the apparent sizes of the centre circles and using the surround circles to determine response, ie by selecting the side with the larger surrounds. If they did so, however, then accuracy would be low in the condition where the larger target was surrounded by smaller circles. All subjects had high accuracy in this condition, however, so we can be confident that their responses were being driven by the apparent sizes of the centre circles, and not by their surrounds.

If subjects selected the side with the centre circle that looked larger, as requested, then they would be highly accurate when context made the difference easy to see and highly inaccurate when it made the difference seem to be in the opposite direction. All but two of the sixty-four subjects responded in this way. Two responded in exactly the opposite way, however; when the real difference was easy to see they selected the side with the smallest centre circle. That was therefore taken to be the correct response for these two subjects.

Every one of the sixty-four subjects was context-sensitive. This is shown most clearly by accuracy when the real size difference was $2 \%$, as in figure 1 . All subjects then performed much more accurately when context made the difference easier to see (mean: 15.5 correct out of 16 trials) than when it made it harder to see (mean: 1 correct out of 16 trials). Any individual differences within this population therefore involve the strength of context sensitivity, not its presence or absence.

The relation between accuracy and actual size difference for the conditions where the large centre circles appeared within larger surrounds is given in figures 2 and 3 . As expected, this shows that accuracy is very low when the real size difference is $2 \%$ and high when it is $18 \%$. We can be confident that errors in these conditions depend upon context because when the context is the other way round, ie with larger targets in small surrounds, performance is near $100 \%$ for all size differences used. Accuracy when size contrast impairs discrimination therefore provides a specific measure of context sensitivity. Context sensitivity can be efficiently quantified by calculating the total number of correct responses out of 80 across all five size differences combined. This measure increases as context sensitivity decreases. There were wide individual differences in these scores, with the highest being 68 out of 80 (a male mathematician), 

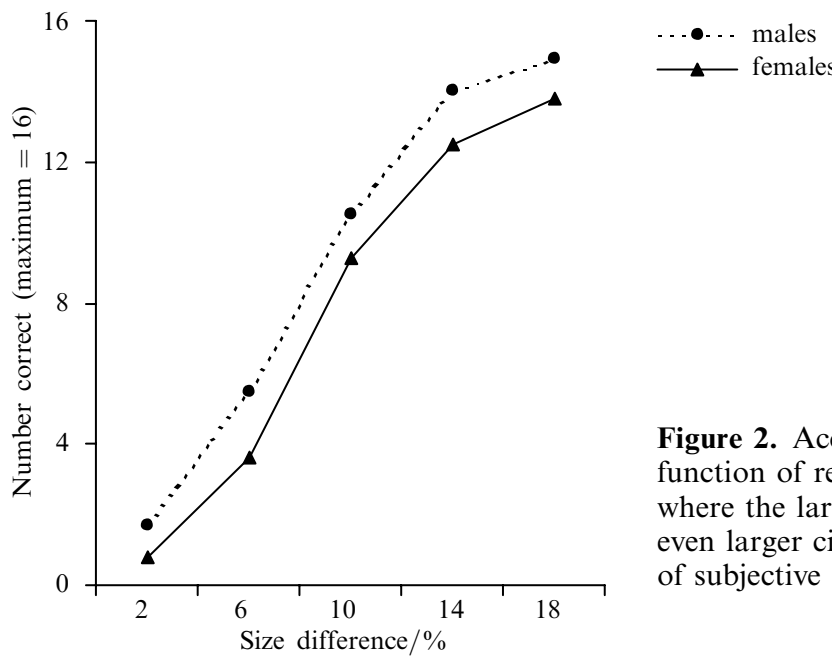

Figure 2. Accuracy for males and females as a function of real size difference for the conditions where the larger centre circle was surrounded by even larger circles. Size contrast moves the point of subjective equality from $0 \%$ to around $8 \%$.
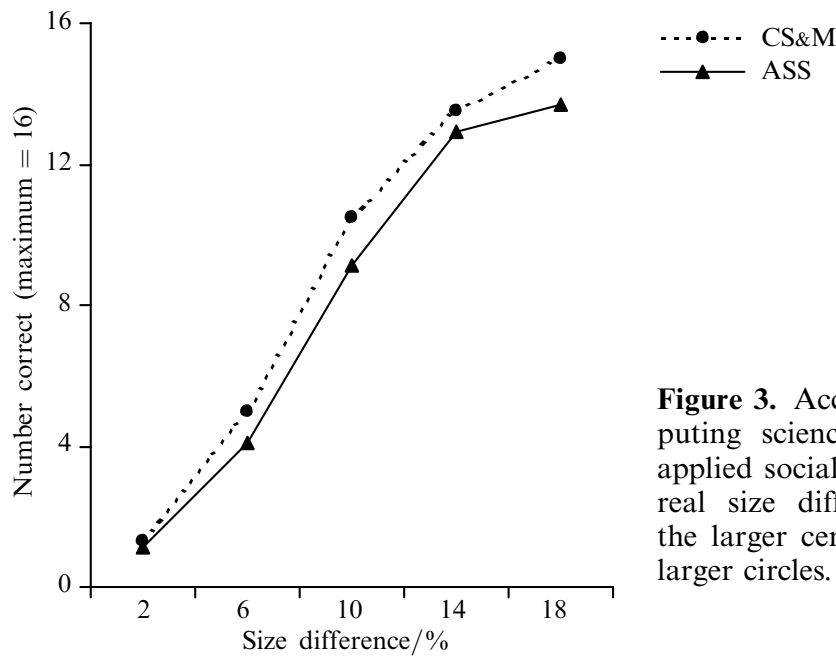

Figure 3. Accuracy for those working in computing science and mathematics (CS\&M) and applied social science (ASS) as a function of the real size difference for the conditions where the larger centre circle was surrounded by even larger circles.

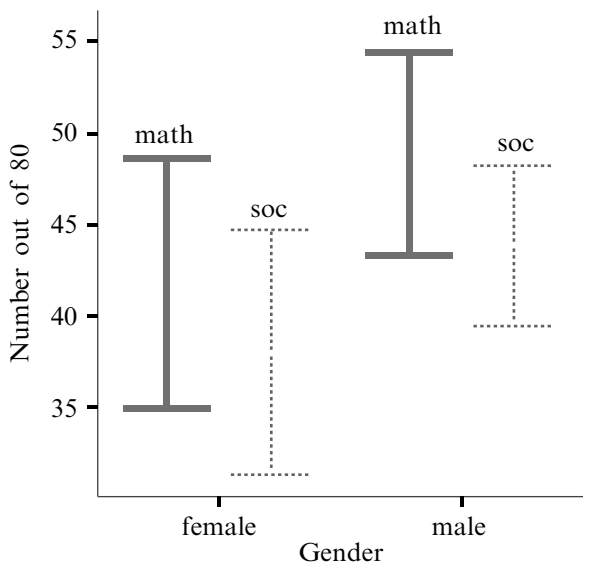

Figure 4. Means and confidence intervals for accuracy over all conditions in which the larger centre circle is surrounded by even larger circles. High scores imply low context sensitivity. Mathematicians and computer scientists are shown as 'math' and applied social scientists as 'soc'. (Note: Error bars show $95.0 \% \mathrm{Cl}$ of Mean.)

and the lowest being 14 out of 80 (a female social scientist). This therefore provides a measure that is both specific and sensitive. Means and confidence intervals for this measure as a function of sex and academic discipline are given in figure 4. 
To analyse group differences, a three-way ANOVA was run with the following results: for sex, $F_{1,7}=5.261, p=0.026$; for academic discipline, $F_{1,7}=2.84, p=0.128$; for academic status, $F_{1,7}=1.418, p=0.239$. None of the interactions approached significance. Planned independent sample $t$-tests were also used to analyse the effects of sex and discipline, with the following results: for sex, $t_{62}=2.323, p$ (one-tailed) $=0.012$; for discipline, $t_{62}=1.528, p$ (one-tailed) $=0.066$.

Figure 4 shows that there is overlap in the strength of context sensitivity between all four groups. However, this overlap is less at the extremes, where subjects tend to have the predicted sex and profession. For example, five of the sixteen male mathematicians have an accuracy in the top $20 \%$ but only one is in the bottom $20 \%$. They therefore tend to have low context sensitivity. Of the sixteen female social scientists only one has an accuracy in the top $20 \%$, but five have an accuracy in the bottom $20 \%$. They therefore tend to have high context sensitivity. Applying Fisher's Exact Test to this difference in distribution shows that $p$ (one-tailed) $=0.04$.

\section{Discussion}

These results provide evidence for the hypothesis that size perception is on average less context-sensitive in males than in females. The results also support other evidence indicating that males tend to be less context-sensitive than females (Voyer et al 1995; Baron-Cohen 2002), and extend it to include a highly specific psychophysical measure of context sensitivity. They provide only weak evidence for the hypothesis that mathematicians and computer scientists tend to be less context-sensitive than social scientists (Baron-Cohen et al 2001).

Our evidence for a sex difference in context sensitivity does not specify its aetiology. A genetic contribution is plausible, however. The paradigm we described provides a suitable means for investigating this issue, as it provides a measure that is specific, sensitive, easy to understand, and takes only a few minutes to apply. The paradigm could also be useful in studies of psychopathologies in which Gestalt perception is impaired, such as schizophrenia (Phillips and Silverstein 2003; Uhlhaas 2003; Uhlhaas et al 2003). It could also be used to help resolve the debate concerning the presence or absence of reduced context sensitivity in autism (Happé 1996; Ropar and Mitchell 1999).

The Ebbinghaus illusion has also been used to study the proposed distinction between dorsal and ventral visual systems (Milner and Goodale 1995). The dorsal stream is that linking visual cortex to posterior parietal cortex, and is thought to be particularly concerned with the control of attention and action. The ventral stream is that linking visual cortex with inferotemporal cortex, and is thought to be particularly concerned with the conscious perception of objects. There is evidence that the dorsal stream is less context-sensitive than the ventral stream (eg Aglioti et al 1995), but this evidence is equivocal and much debated (Bruno 2001; Carey 2001; Franz 2001; Milner and Dyde 2003). If the dorsal stream is less sensitive to context, then the above evidence that males are less sensitive to context raises the possibility that action-directed processing may have a more dominant role in males. This possibility could be tested by looking for male-female differences with the use of the various paradigms developed to distinguish between the putative dorsal and ventral visual systems.

Milner and Dyde (2003) have argued that it is only regions in the depth of the ventral processing stream that are context-sensitive. This seems to conflict with the argument for contextual interactions, with which we began, that implied that local ambiguities are ubiquitous and that context plays a major role in disambiguating them. If so, then contextual interactions are expected in both streams and prior to the division of processing into those streams. This apparent conflict could be resolved by assuming that it is not contextual interaction in general that is limited to the ventral stream, but 
only certain specific contextual interactions, including those producing size contrast in the Ebbinghaus illusion.

We did not find any large differences in context sensitivity between mathematicians/ computer scientists and social scientists. The results do suggest, however, that the former may be less context-sensitive. Large differences would be surprising, given the many similarities between those working within the same academic institution. BaronCohen et al (2001) developed a self-report measure of traits associated with the autistic spectrum, and found that winners in the UK Mathematics Olympiad scored more highly on this scale than control subjects. If this scale reflects a general processing style that includes perception (Baron-Cohen 2002; Happé 1996), then such subjects will on average have low context sensitivity in our size-perception paradigm. This prediction remains to be tested.

Neither time of day nor the point in the women's menstrual cycles were controlled in our study. As diurnal and menstrual hormonal changes affect various cognitive functions (Hampson 1990; Hausmann et al 2000; Sanders et al 2002), this lack of control could have added some noise to the measures obtained. This did not prevent us finding significant differences between males and females, but it may be the case that such differences are greater at some points in the menstrual cycle than at others. The paradigm introduced here is well suited to a test of this possibility.

Finally, the effects of surrounds on size perception may help further clarify our concept of 'context'. We think of contextual interactions as a special class of interactions that do not themselves drive activity but which modulate the effects of those that do. From this point of view it is not adequate to think of context as whatever is not called the target in some perceptual task. Psychophysical methods for distinguishing between driving and contextual interactions have been formally specified by Smyth et al (1996) who used measures of condition mutual information (Kay et al 1998; Phillips et al 1995). In a study of the interaction between cues to texture boundaries, Phillips and Craven (2000), using these methods, found that different cues to coincident texture boundaries do not modulate each other, but simply combine to form the perception of a single texture boundary. For further discussion of this functional concept of context see Kubovy and Cohen (2001), Phillips (2001), and Phillips and Silverstein (2003). In relation to the effects of surrounds on size perception, it is possible that assimilation arises from driving or summative interactions (Stuart et al 1993). In the case of size contrast as studied above, however, responses are driven by the apparent sizes of the centre circles, and not by their surrounds. Surrounds do have an effect, however, so this must be modulatory. This argument implies that context sensitivity is reflected by size contrast but not by assimilation. If so, this suggests the possibility that sex differences in the effects of surrounds on size perception apply to contrast but not assimilation. This hypothesis remains to be tested.

\section{References}

Aglioti S, Goodale M A, DeSouza J F X, 1995 "Size contrast illusions affect the eye but not the hand" Current Biology 5 679-685

Baron-Cohen S, 2002 "The extreme male brain theory of autism" Trends in Cognitive Sciences 6 $216-222$

Baron-Cohen S, Richler J, Bisarya D, Gurunathan N, Wheelwright S, 2003 "The systemizing quotient: an investigation of adults with Asperger syndrome or high-functioning autism, and normal sex differences" Philosophical Transactions of the Royal Society of London, Series B 358 $361-374$

Baron-Cohen S, Wheelwright S, Skinner R, Martin J, Clubley E, 2001 "The autism-spectrum quotient (AQ): Evidence from Asperger syndrome/high-functioning autism, males and females, scientists and mathematicians" Journal of Autism and Developmental Disorders 31 - 17 
Briskman J, Happé F, Frith U, 2001 "Exploring the cognitive phenotype of autism: Weak 'central coherence' in parents and siblings of children with autism: II. Real-life skills and preferences" Journal of Child Psychology and Psychiatry 42 309-316

Bruno N, 2001 "When does action resist visual illusions?" Trends in Cognitive Sciences 5 379-382 Carey D P, 2001 "Do action systems resist visual illusions?" Trends in Cognitive Sciences 5 109-113 Franz V H, 2001 "Action does not resist visual illusions" Trends in Cognitive Sciences 5 457-459

Hampson E, 1990 "Variations in sex-related cognitive abilities across the menstrual cycle" Brain and Cognition $1426-43$

Happé F G E, 1996 "Studying weak central coherence at low levels: Children with autism do not succumb to visual illusions. A research note" Journal of Child Psychology and Psychiatry $37873-877$

Happé F, 1999 "Autism: cognitive deficit or cognitive style?" Trends in Cognitive Sciences 3248 - 254

Happé F, Briskman J, Frith U, 2001 "Exploring the cognitive phenotype of autism: Weak 'central coherence' in parents and siblings of children with autism: I. Experimental tests" Journal of Child Psychology and Psychiatry 42 299-307

Hausmann M, Slabberkoorn D, Van Goozen S H M, Cohen-Kettenis P T, Gunturkun O, 2000 "Sex hormones affect spatial abilities during the menstrual cycle" Behavioral Neuroscience $1141245-1250$

Kay J, Floreano D, Phillips W A, 1998 "Contextually guided unsupervised learning using multivariate local processors" Neural Networks 11 117-140

Kubovy M, Cohen D J, 2001 "What boundaries tell us about binding" Trends in Cognitive Sciences $593-95$

Milner D, Dyde R, 2003 "Why do some perceptual illusions affect visually guided action, when others don't?" Trends in Cognitive Sciences $710-11$

Milner D, Goodale M A, 1995 The Visual Brain in Action (Oxford: Oxford University Press)

Phillips W A, 2001 "Contextual modulation and dynamic grouping in perception" Trends in Cognitive Sciences $595-97$

Phillips W A, Craven B, 2000 "Interactions between coincident and orthogonal cues to texture boundaries" Perception \& Psychophysics 62 1019-1038

Phillips W A, Kay J, Smyth D, 1995 "The discovery of structure by multi-stream networks of local processors with contextual guidance" Network: Computation in Neural Systems 6 225-246

Phillips W A, Silverstein S M, 2003 "Convergence of biological and psychological perspectives on cognitive coordination in schizophrenia" Behavioral and Brain Sciences 26 65-82

Phillips W A, Singer W, 1997 "In search of common foundations for cortical computation" Behavioral and Brain Sciences $20657-722$

Ropar D, Mitchell P, 1999 "Are individuals with autism and Asperger's syndrome susceptible to visual illusions?" Journal of Child Psychology and Psychiatry $401283-1293$

Sanders G, Sjodin M, de Chastelaine M, 2002 "On the elusive nature of sex differences in cognition: Hormonal influences contributing to within-sex variation" Archives of Sexual Behavior 31 $145-152$

Smyth D, Phillips W A, Kay J, 1996 "Measures for investigating the contextual modulation of information transmission" Network: Computation in Neural Systems $7307-316$

Stuart G W, Bossomaier T R J, Johnson S, 1993 "Preattentive processing of object size: implications for theories of size perception" Perception 22 1175-1193

Uhlhaas P, 2003 Gestalt Perception in Schizophrenia Spectrum Disorders PhD thesis, Department of Psychology, University of Stirling, Stirling, UK

Uhlhaas P, Silverstein S M, Phillips W A, 2003 "Evidence for impaired visual context processing in thought disordered schizotypes" Schizophrenia Research (in press)

Voyer D, Voyer S, Bryden M P, 1995 "Magnitude of sex differences in spatial abilities: A metaanalysis and consideration of critical variables" Psychological Bulletin 117 250-270

Witkin H A, Lewis H B, Hertzman M, Mackover K, Meissner P B, Wapner S, 1954 Personality through Perception (New York: Harper \& Brothers) 


\section{PERCEPTION}

VOLUME 332004

www.perceptionweb.com

Conditions of use. This article may be downloaded from the Perception website for personal research by members of subscribing organisations. Authors are entitled to distribute their own article (in printed form or by e-mail) to up to 50 people. This PDF may not be placed on any website (or other online distribution system) without permission of the publisher. 